\title{
TRAFFIC CONTROLLED-DEDICATED SHORT RANGE COMMUNICATION: A SECURE COMMUNICATION USING TRAFFIC CONTROLLED DEDICATED SHORT RANGE COMMUNICATION MODEL IN VEHICULAR AD HOC NETWORKS FOR SAFETY RELATED SERVICES
}

\author{
${ }^{1}$ K. Chandramohan and ${ }^{2} \mathbf{P}$. Kamalakkannan \\ ${ }^{1}$ Department of Computer Science and Engineering, Gnanamani College of Engineering, Namakkal, Tamilnadu, India \\ ${ }^{2}$ Department of Computer Science, Government Arts and Science College (Autonomous), Salem-7, Tamilnadu, India
}

Received 2014-01-31; Revised 2014-02-12; Accepted 2014-03-01

\begin{abstract}
To increase the road safety and secure communication among the vehicles in the network environment, Dedicated Short Range Communication (DSRC) is followed. Nowadays, research over DSRC is dramatically increased for enhancing the road safety applications. The main task of DSRC is to protect the vehicles by communicating the warning message regarding the vehicle changing conditions, traffic occurrence and dangers over the road in the network. So, it is necessary to maintain the accurate communication timely with high reliability by implementing the appropriate protocol. In the literature there are several methods which provided specifications defined in the physical layer and the Medium Access Control (MAC) layer. In those methods, current IEEE 802.11p MAC is not able to provide predictable Quality of Service (QoS) for high-priority safety services. Motivated by the fact that the existing work provided three levels of safety-related broadcast services, but did not focus on current traffic load conditions, in this study, we plan to present Traffic Controlled DSRC (TC-DSRC) model to analyze and categorize the traffic patterns in the vehicular communication for safety related application. A new algorithm is presented to evaluate the process of traffic controlled DSRC model for secure communication in VANET. The dedicated short range communication broadcast of messages is sent to all the vehicles at a specific instance for defined radius on the traffic zone. Multiple traffic load conditions are categorically stated to handle the vehicular safety with quick response time. Integrating these two performance metrics (i.e., quick response time and security), by using the proposed algorithm, the traffic patterns are categorized for communication between vehicles to provide the safety measure. In addition, our proposed scheme with the categorization using traffic patterns improves the network performance by deriving a specific pattern. Compared with the recent standard of $802.11 \mathrm{p}$ wireless vehicular networks the scheme proposed in this study is more robust and operate under vehicle-to-vehicle mode in order to address the secure communication between the vehicles. The effectiveness of secure communication in vehicular ad hoc network with varied vehicular speed and changing network topology is evaluated numerically using realistic simulation data obtained from traffic engineering systems.
\end{abstract}

Keywords: VANET, Communication, Security, DSRC Model, IEEE 802.11p MAC, Traffic Controlled DSRC Model.1

\section{INTRODUCTION}

In recent years, the increase in population over human, passenger and freight transport have made the road traffic to be analyzed accordingly. The Vehicular
Ad hoc Networks (VANETs) are comprehensive with numerous facts. Hao et al. (2011), the author proposed an isolated key association construction mechanism that sustained on group in Vehicular Ad hoc Networks (VANETs). Isolated key organization is expected to Corresponding Author: K. Chandramohan, Department of Computer Science and Engineering, Gnanamani College of Engineering, Namakkal, Tamilnadu, India 
formulate promising revocation in spite of huge vehicles, compared with the middle key association assumed by the existing group inscription schemes.

Besides the process of achieving certain task utilities in the network environment, consistent data provisioning is one of the other serious concern. A precise weather description may prevent accidents in a practical manner by different nodes/drivers. For this kind of information, a novel type of information expertise termed Vehicular Ad-hoc Network (VANET) is being enhanced. Intrusion detection technique laid different level of challenges for the efficient implementation of VANETs. Pattnaik and Pattanayak (2014) analyzed different attacks that in VANETs and to mitigate it, watchdog technique was presented for efficient detection of intrusion in VANET.

As a key purpose of the reputation of privacy in Vehicular Ad hoc Networks (VANETs), position privacy is highly required in VANETs to totally increase the security and privacy. Even though widespread alias changeable presents a skilled decision for position isolation in VANETs, if the pseudonyms are unclear in an offensive point or position, such an elucidation might turn into offensive. The designing of Delay Tolerant Network (DTN) operate in an environment where there provisioning is not provided for end to end path between source and destination. An effective buffer management mechanism and combined with epidemic routing for supporting delivery of realtime information using indexer, scheduler and dropper to perform the buffer management.

The IEEE 802.16 Cicconetti et al. (2010) supports mobile BWA in a fully fledged manner, also defined power saving functions at the Medium Access Control (MAC) layer, which are designed and can be operated during open traffic sessions for minimizing the energy consumption. In addition, the previous models of IEEE 802.11 DCF cannot be applied as they assume that the propagation time can be neglected. The study by Simo et al. (2010) studies the impact of the distance on the behavior of IEEE 802.11 DCF and presents an analytical model of IEEE 802.11.

To supervise with the question, in (Lu et al., 2012), presented an efficient also called varying at Social Spots (PCS) technique to design a position privacy to achieve security in hand to hand. Shim (2012), the author presented a conditional isolation preserving support method, termed as CPAS, vehicle-to-infrastructure transportations in vehicular ad hoc networks.

Tseng et al. (2011), Reed-Solomon codes (RS-codes) are engaged to manufacture an encouragement system by examining one detached logarithm demonstration crisis. The study (Mershad et al., 2013) presented a mechanism that uses the advantage of RSUs that are connected to the
Internet and subsequently provide various types of information to VANET users. A VANET-based Ambient Ad-Dissemination scheme (VAAD) (Li et al., 2013) was provided that supports secure ad disseminations with pragmatic cost and effect control.

DSRC based Vehicular Ad Hoc Network (VANET) technique turn out to be significant impact for vehicular security. Ma et al. (2012), presented an authenticated and distributive cross-layer system for maintaining table of straight channel in DSRC with three phases of transmits services that are severe to most possible vehicle-safety-related applications.

The author Ros et al. (2012) presented a broadcast algorithm for vehicular scenarios employing local information through periodic beacon messages. It also waits for a shorter period of time before retransmission. During time-out expiration, vehicle retransmits if it is aware of at least one neighbor. Routine status messages are launched occasionally to bordering vehicles to notify them of the present condition of the initiating vehicle (e.g., position speed, path), whereby the getting vehicles/drivers can then expect any possible exposures (e.g., traffic jam ahead) and obtain essential action. Event-driven safety messages are activated by quick alterations in vehicle actions for instance a hard brake or an airbag detonation. To facilitate preventive action, it is necessary that both kinds of safety messages being established properly by surrounding vehicles in an appropriate fashion. Zhang et al. (2008) presented TESLA, a mystification based protocol, to decline the calculation overhead.

In security driven with frequent message communication, it is large to set protocols with diminutive calculation simplicity for appropriate and unfailing message processing. In this study, a Traffic Controlled based DSRC (TC-DSRC) model is implemented to enhance the road safety applications. Based on the defined radius on the traffic zone over the VANET, the traffic patterns are categorized in the vehicular communication at the specified instance. The contributions of TC-DSRC model are:

- To present traffic controlled DSRC model to analyze and categorize traffic patterns in VANET for safety related applications

- To design dedicated short range communication broadcast model and sent messages to all the vehicles with defined radius on the traffic zone

- To categorize and handle multiple traffic load conditions with quick response time

The rest of this study is structured as follows: Section 2 discusses the techniques of Traffic Controlled DSRC model for enhancing the road safety applications, 
correspondingly. Section 3 presents experimental setting and section 4 represents the results for evaluating the proposed TC-DSRC model. Finally, Section 5 gives concluding remarks.

\section{TRAFFIC CONTROLLED DSRC}

In this section a communication in VANET using Traffic Controlled DSRC is introduced. Then the system model is presented followed by TC-DSRC model.

\subsection{Communication in VANET using Traffic Controlled DSRC}

Dedicated Short Range Communications (DSRC) was designed for short-to-medium range communication satisfying a specific set of standards and protocols. DSRC is a wireless technology which processed with 5.9 $\mathrm{GHz}$ range with $75 \mathrm{MHz}$ of spectrum. It is a wireless procedural network to process the $\mathrm{V} 2 \mathrm{~V}$ (vehicle-tovehicle) communication. The main objective of DSRC is to design safety vehicular application to minimize occurrence of accidents on the road. DSRC contains six set of service and one control channel. The six set of service channels are used for non-safety crucial messages whereas control channel is utilized for sending and receiving warning or safety messages.

Two types of DSRC devices are utilized for vehicular safety communication in VANET, namely (i) On-Board Unit (OBU) and (i) Road-Side Unit (RSU). Communication devices mounted on vehicle is referred to as the On-Board Unit (OBU) whereas communication units located outside the roads are called as road-side unit (RSU). DSRC includes two types of operational models, namely (i) roadside-to-vehicle and (2) vehicleto-vehicle. In the proposed system, the traffic controlled DSRC operate under vehicle-to-vehicle mode to address security communication among vehicles.

To achieve secure communication in VANET, traffic controlled DSRC model initially analyzes the set of vehicles in road signal and the process of sorting vehicles are performed at the traffic signals correspondingly. To categorize the traffic patterns in traffic signals, DSRC model forwards the message to all the adjacent vehicles in the network environment based on the defined radius on the traffic zone.

Figure 1 shows the flow diagram of the proposed TC-DSRC model. Secure communication in TCDSRC model is achieved by analyzing the traffic patterns, monitoring the neighboring vehicles followed by the categorization of traffic patterns based on the radius in the network environment.

\subsection{System Model}

Assume a setup of VANET framework with vehicleto-vehicle communications for exchanging information on a highway. The highway comprises of number of lanes with vehicles processing in individual directions. In the VANET framework, the subsequent assumptions are made. Vehicles on the highway can be symbolized as a compilation of arbitrary and statistically indistinguishable locations in one dimensional network environment. The communication range for every location is identical, deterministic and indicated by R. Data packets are received at each station based on the Poisson procedure with rate $\lambda=300$ packets $/ \mathrm{min}$. The collision possibility of the packet data arrived at each station is stable in spite of different traffic state. Channel conditions are perfect inside radius $\mathrm{R}$, packet loss happens exclusively as an effect of packet impacts and collisions at the rear-end packets.

When the network topology does not changes considerably for one packet communication time, space among lanes on main road is insignificant compared to the time-span of the network. Let VD be a moving vehicle in road network measured in $\mathrm{km}$ on the highway. The average number of vehicles in the communication model of the labeled vehicle $(\mathrm{Nt})$ counting the marked station is calculated as Equation 1:

$\mathrm{Nt}=1+2 \mathrm{VD} * \mathrm{R}$

Although an unlimited buffer size is noticeably, it might be sensible in safety applications as there should be not numerous packets coming up to be transmitted and a packet containing stale content should be restored by a novel one with the most contemporary information. 2.3Traffic Controlled DSRC (TC-DSRC) Model

The traffic controlled DSRC (TC-DSRC) model analyzes and categorizes the traffic patterns in vehicular communication for safety related application. The dedicated short range communication broadcast of messages is done in specific instance for defined radius on the traffic zone. The process of TC-DSRC model is explained in Fig. 2.

The TC-DSRC model follows subsequent steps to achieve secure communication and high reliability, namely:

- Monitoring the neighboring vehicles

- Categorization of traffic patterns

- Broadcasting the message under defined radius

- Handle the vehicular safety 


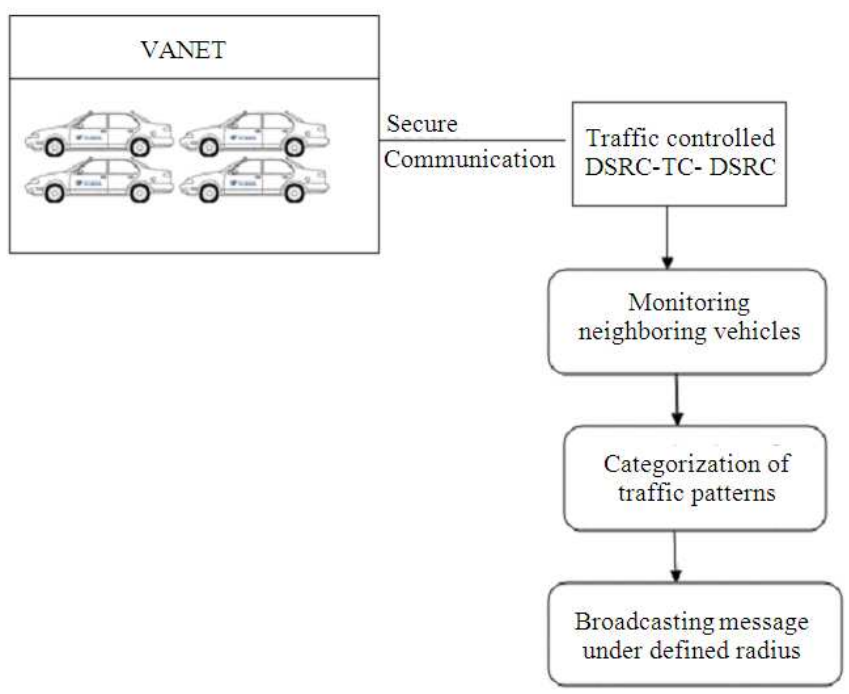

Fig. 1. Flow diagram of the proposed TC-DSRC model

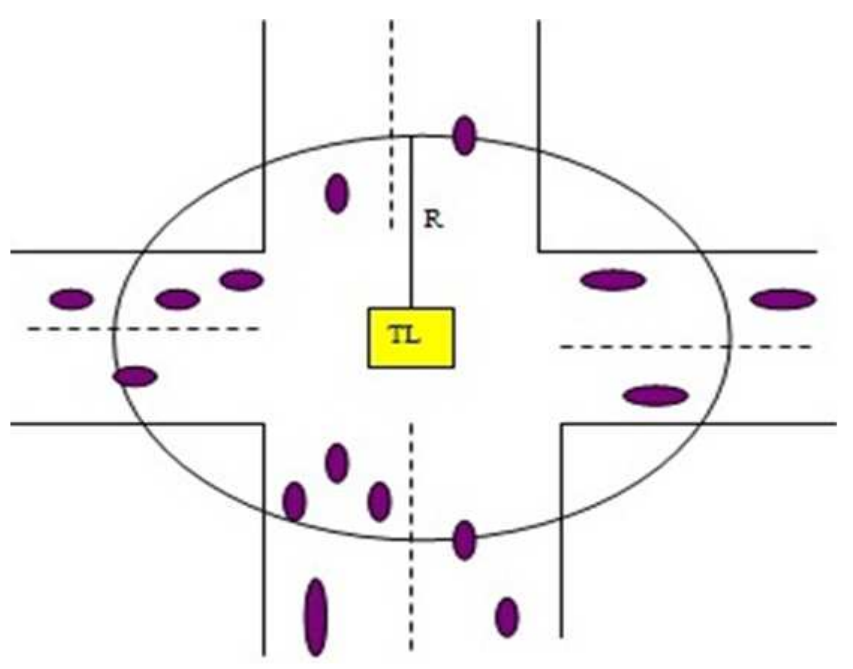

Fig. 2. Traffic Controlled DSRC model

\subsubsection{Monitoring the neighboring vehicles}

The first step in TS-DSRC consists of monitoring the neighboring vehicles in the VANET in order to achieve communication in more efficient manner. At this juncture, it is highly significant to maintain reliable authorized moving vehicles on road. The neighboring vehicles are identified by sending HELLO packet data and each vehicle maintains neighbor vehicle table with necessary information consistent with packet type. TCDSRC utilizes neighbor table that allocates an entry for every neighboring node, which comprises information associated to node including the location of vehicle, estimated waiting time, estimated transmission delay, required transmission energy and estimated packet delivery ratio as illustrated in Fig. 3.

The three concluding parameters estimated transmission delay, transmission energy and packet delivery ratio are expected by neighbor manager, whereas parameters namely vehicle location and estimated waiting time are expected by adjacent nodes utilizing their individual neighbor managers. The packet structure is modified based on the HELLO packet received by the nodes. Each vehicle in the road network forwards a HELLO message with the location, remaining energy and its evaluation of the other set of vehicles. 


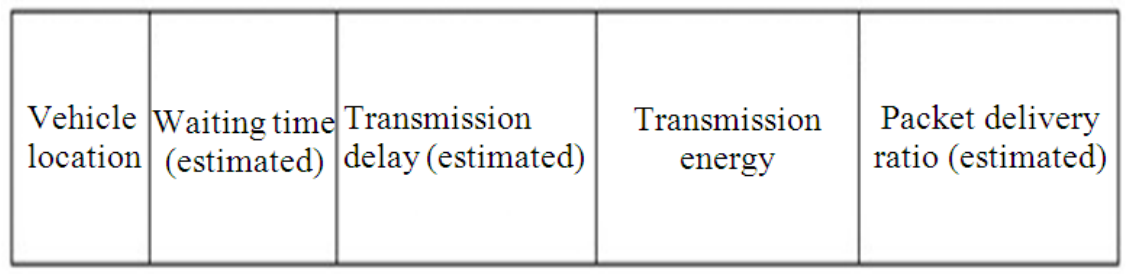

Fig. 3. Packet structure

Neighboring nodes use HELLO packets to acquire existing entries of the node, adjoining nodes that progress with inside the node's locality. The neighboring nodes at the same time also remove the entries when adjacent nodes travel away or break down, which can be noticed in case of not receiving the HELLO packets after a period of time (timeout).

\subsubsection{Categorization of Traffic Patterns}

The second step in TC-DSRC is categorization of traffic patterns. The traffic-actuated controller functions by adjusting volume of vehicles on the road. This method does not set any timing for the transmission of signals, but provides the volume of vehicles on the road at one specified instant and then design the occurrence and interval of traffic lights consequently. The trafficactuated has three essential components:

- Detector

- Controller unit and

- $\quad$ Signal heads (traffic lights)

The detector in TC-DSRC identifies the occurrence and volume of vehicles on the road. The controller unit in TC-DSRC acts as the brain of the system, as it acquires data from detector and selects the frequency and period of consecutive signal cycle. Finally the signal heads in TC-DSRC provide visual demonstration to drivers by revealing lights in three different colors red, yellow and green.

Consequently, instead of calculating a traffic pattern by daily flow profiles on all communication, it is simple to derive a pattern by daily traffic flow profiles based on key locations that are preferred on the basis of their operation over the network environment.

The traffic patterns $(\mathrm{P})$ in the specified location is determined as Equation 2:

$\mathrm{TP}_{\mathrm{d}}=\left(\mathrm{VV}_{\mathrm{n}, \mathrm{sl}}, \ldots, \mathrm{V}_{\mathrm{n}, \mathrm{sn}}\right)$

where, ' $\mathrm{TP}_{\mathrm{d}}$ ' denotes the traffic pattern followed for a specific day of week ' $d$ ' at a specified location ' $s 1$ ' for a vehicle ' $\mathrm{V}_{1}$ '. Now, the categorization of the traffic patterns is performed based on the density of the vehicles across the specified path. The density of the traffic patterns are determined as Equation 3:

$$
\mathrm{VD}_{\mathrm{i}}=\mathrm{TOtV}_{\mathrm{i}} / \mathrm{t}
$$

where, the vehicle Density ' $\mathrm{VD}_{\mathrm{i}}$ ' is represented by the total number of vehicles flows in the specified roadway ' $\mathrm{TOtV}_{\mathrm{i}}$ ' per specific time interval ' $\mathrm{t}$ '.

At the same time the categorization is also performed based on the occurrence of patterns over a set of traffic data Equation 4:

$$
\mathrm{C}_{\mathrm{i}}=\left\{\mathrm{d} \in \mathrm{D}_{\mathrm{S} 1} \mid \mathrm{k}(\mathrm{d})=\mathrm{k}\right\}
$$

where, the categorization for a cluster ' $C_{i}$ ' is represented for the specific days of week ' $d$ ', followed for a specified location ' $\mathrm{D}_{\mathrm{S} 1}$ ' per the function ' $\mathrm{k}(\mathrm{d})$ ' that allocates the required patterns into the appropriate network.

The categorization of traffic patterns in TC-DSRC is performed on the occurrence of traffic over the days of week. The categorization of traffic patterns are performed as follows in TC-DSRC:

- Category 1: Monday, shows a high traffic volume while morning at all locations but relatively equal traffic flow occurred at evening

- Category 2: Tuesday, Wednesday, Thursday, Friday morning shows a regular set of traffic flow at the specified locations

- Category 3: Friday evening shows high volume of traffic occurrence

- Category 4: Saturday and Sunday represents low volume of traffic across the network environment

Based on the above assumptions, traffic patterns are analyzed and processed based on the volume of traffic over the network environment. 


\subsubsection{Broadcasting the Message Under Defined Radius}

Once the analysis and categorization of traffic patterns are performed using TC-DSRC, the warning message regarding the traffic are sent to nearby vehicles by other set of vehicles to secure flow in the network environment in order the communication to be secure. To provide a secure communication, in TCDSRC, a radius across the traffic signals is determined. The vehicles which are present within the determined radius receive the occurrence of traffic message and the message is propagated to all vehicles in the network environment.

While sending this warning message to all set of vehicles in VANET, traffic load conditions are restricted by directing other vehicles in other directions to reduce traffic flow at the busy locations. The Fig. 2 describes the process of defining the radius for the communication between he vehicles in the specified instant. The pseudo code below describes the entire process of traffic controlled DSRC model for secure communication in VANET.

Input: Number of vehicles V, VD (Vehicle Density), message M, Pattern P, Category (C), users U, time t, specified location sl

Begin

Identify the $\mathrm{V}$ in the $\mathrm{sl}$

Determine the VD in sl

Identify the neighboring vehicles to $\mathrm{V}$

Maintain a neighbor table with the position, packet transmission speed and distance

Calculate P using Equation 2

Sort the traffic patterns

Categorize the traffic flow based on $\mathrm{P}$

Set min (VD) to all the sl

If $\mathrm{V}>\min (\mathrm{VD})$

Identify the neighboring vehicles

Pass the $\mathrm{M}$ to all the vehicles

Redirect the path to reduce the traffic flow Else

Traffic flows based on pattern categorization End If

End

The process of TC-DSRC starts with initialization of the number of vehicles ' $\mathrm{V}$ ' at a specified location ' $s$ ' ' in the environment. Next, the vehicle density 'VD' is identified for specified location 'sl'. The third step involved in TC-DSRC is to identify the neighboring vehicles to the vehicle ' $\mathrm{V}$ '. Then the TC-DSRC maintains neighbor table information regarding position, packet transmission, the speed at which the packet traverse in the network and finally the distance from the vehicle ' $\mathrm{V}$ '. The traffic patterns are identified, by sorting the traffic patterns and categorizing the traffic flow based on the patterns observed.

The minimum vehicle density is then set to all the vehicles in the network. If the distance of vehicle is less than the vehicle density then the neighboring vehicles is identified, the message is passed to the vehicles and finally the path is redirected to reduce the traffic flow. In this way, the above said processes are followed to normalize the multiple traffic load conditions at a specified path to handle the vehicular safety.

\section{EXPERIMENTAL EVALUATION}

Experiments are conducted in a vehicular ad hoc network with varied vehicular speed and changing network topology to evaluate the performance and suitable of proposed traffic controlled DSRC model using the Table 1 given below and compared against DSRC scheme in the recent standard of $802.11 \mathrm{p}$ wireless vehicular networks.

Consider a highway situation with two lanes in every direction. Vehicles are located consistently on the road and journey at speed of $30 \pm 5 \mathrm{~m} / \mathrm{s}$ (roughly equivalent to the range of $56-80 \mathrm{miles} / \mathrm{h}$ ). The highway location gives us the expediency to assess the inferior bound of the performance, by organizing vehicles with senior speeds and superior densities to shove several units into a high-load situation. The characteristic of city road scenario are processed with the settings, where the key allocation performance is certainly much better than that under a high-load road condition. The performance of the proposed traffic controlled DSRC model is evaluated in terms of vehicle density, packet reception delay and vehicle traffic load.

Table 1. Parameter of analysis

\begin{tabular}{|c|c|}
\hline Spectrum & $75 \mathrm{MHz}$ \\
\hline Channels & $\begin{array}{l}\text { Seven channels } \\
\text { (One-control channel/ } \\
\text { six-service channel) }\end{array}$ \\
\hline Communication range & $300 \mathrm{~m}$ \\
\hline Packet size (bytes) & 200 \\
\hline Message generation & 100 \\
\hline \multicolumn{2}{|l|}{ Interval (msec) } \\
\hline Data range & $6 \mathrm{mbps}$ \\
\hline Broadcast period & $30 \mathrm{~s}$ \\
\hline
\end{tabular}




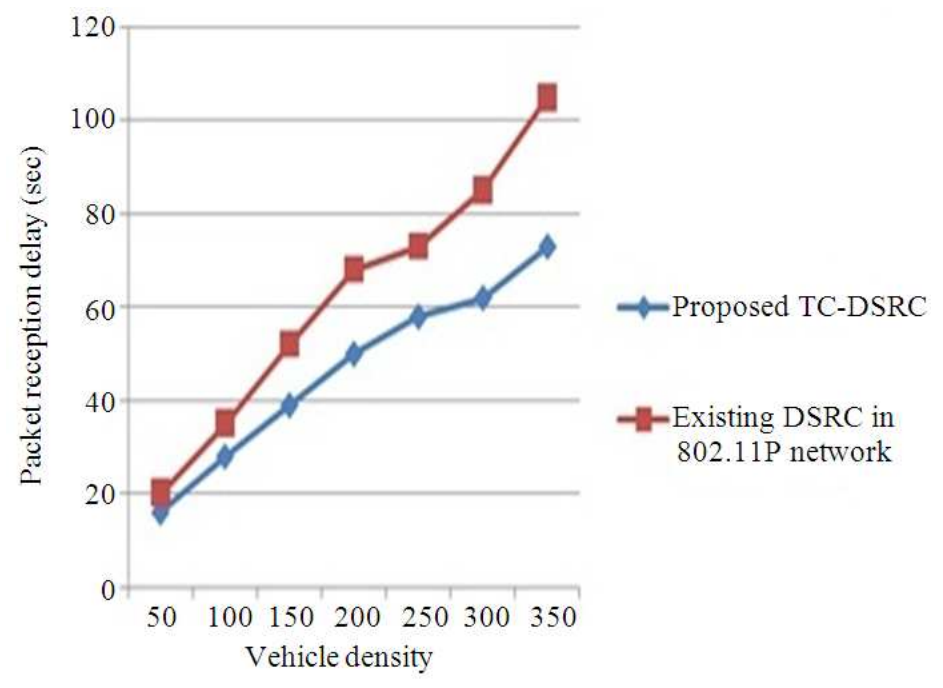

Fig. 3. Measure of packet reception delay

\section{SIMULATION RESULTS}

To evaluate the performance of the proposed traffic controlled DSRC model is estimated and illustrated in the following graphs by comparing the values against the existing DSRC (Ma et al., 2012) scheme in the recent standard of $802.11 \mathrm{p}$ wireless vehicular networks. The proposed TC-DSRC analyzes and categorizes the traffic pattern with high reliability based on the traffic flow observed during different periods. The existing DSRC in 802.11 networks used the control channel to deliver limited classes of safety related measures. Generally speaking, the existing DSRC in 802.11 networks performs well in varied vehicular environments, whereas the proposed TC-DSRC be more suitable for current traffic load and network conditions that vary to a larger extent.

\section{DISCUSSION}

The packet reception delay is measured for a secure communication in VANET based on the vehicle density and compared with the existing DSRC in $802.11 \mathrm{p}$ networks (Ma et al., 2012). Figure 3 describes packet reception delay measured based on vehicle density for secure communication in VANET. Compared to the existing DSRC in 802.11p networks, the proposed TC-DSRC model shows minimum delay in packet reception. This is because the proposed TCDSRC model categorizes traffic patterns based on the days. The traffic flow is properly analyzed and the TC-DSRC model broadcast the message to the set of vehicles which lies within the defined radius. As a result of this the categorization is performed, certain other vehicles redirect through path to reduce the traffic flow. So, the packet reception delay is less in the proposed TC-DSRC model with $20-22 \%$ low in the proposed TC-DSRC model.

The vehicle traffic load is measured and compared with the existing DSRC in $802.11 \mathrm{p}$ networks (Ma et al., 2012). Figure 4 describes vehicle traffic load conditions in VANET based on density of vehicles in road network. Compared to existing DSRC in $802.11 \mathrm{p}$ networks, proposed TC-DSRC model realizes less traffic load. This is because once traffic occurs, the warning messages are sent to nearby vehicles in TCDSRC model. The vehicles receive message only if it lies within desired radius of the traffic load. So, traffic load becomes less by redirecting route path of vehicles in the network with $10-15 \%$ less in the proposed TC-DSRC scheme.

The security over the vehicles and further compared with the existing DSRC in 802.11p networks (Ma et al., 2012). Figure 5 describes the security over the vehicles that are measured based on the vehicle density. As the proposed TC-DSRC model has minimum delay in packet reception and traffic load, the security achieved over the vehicles in the network environment are high compared to the existing DSRC scheme with $10-15 \%$ higher than the existing DSRC model. 


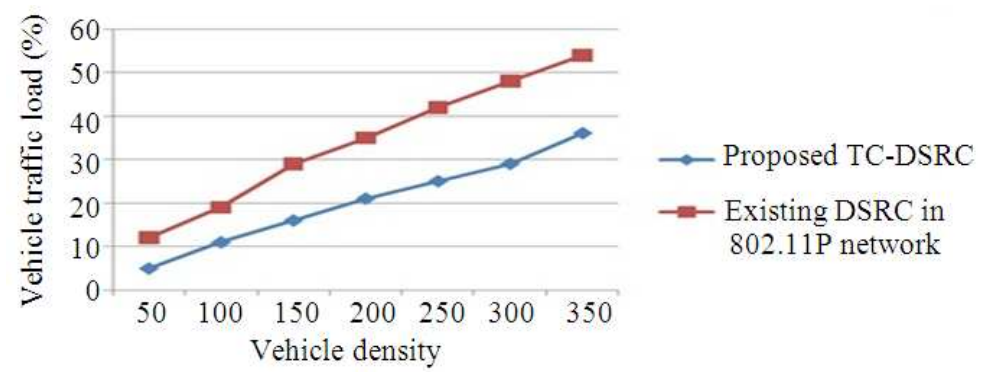

Fig. 4. Measure of vehicle traffic load

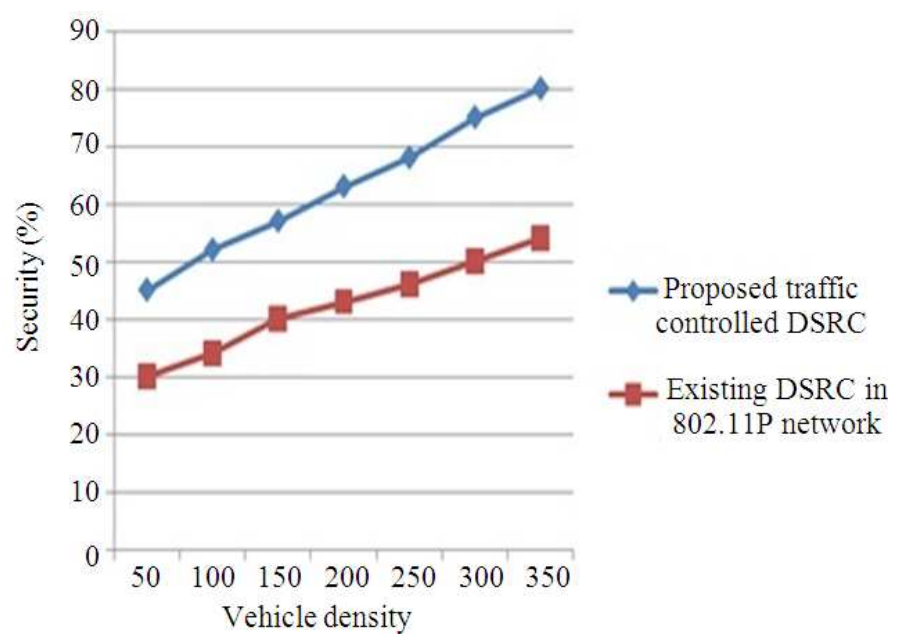

Fig. 5. Vehicle density Vs security

\section{CONCLUSION}

An efficient model TC-DSRC is presented in this work to enhance secure communication in VANET. At first, the traffic over the road network is analyzed and categorized based on the traffic patterns. The traffic patterns are organized based on the traffic flow observed in the specific days. Once categorization is performed effectively, TC-DSRC model transmit the warning messages to all set of vehicles in the road network environment. However a small variations in the categorization pattern further deteriorate the entire network. Therefore measures can be taken to provide mechanisms that can not only be applied for a specific pattern but also for wide range of patterns. Multiple traffic load conditions are categorically stated to handle the vehicular safety with quick response time. Experimental evaluation is carried out to estimate the performance of the proposed TC-DSRC model and reveals that it achieves minimum packet reception delay, less traffic load and high level of security to handle the vehicles. Performance results reveal that the proposed
TC-DSRC model provides $10-15 \%$ high security, with the packet reception delay minimized to $20-22 \%$. Future works will focus on the various routing protocols for DSRC based services in VANET. Although $802.11 \mathrm{p}$ protocol is a major routing protocol in VANET, several other routing protocols are emerging we will evaluate these protocols to further increase the capacity and security of network.

\section{REFERENCES}

Cicconetti, C., L. Lenzini, E. Mingozzi and C. Vallati, 2010. Reducing power consumption with qos constraints in IEEE 802.16e wireless networks. IEEE Trans. Mobile Comput., 9: 1008-1021 DOI: $10.1109 /$ TMC.2010.53

Hao, Y., Y. Cheng, C. Zhou and W. Song, 2001. A distributed key management framework with cooperative message authentication in VANETs. IEEE J. Selected Areas Commun., 29: 616-629. DOI: 10.1109/JSAC.2011.110311 
Li, Z., C. Liu and C. Chigan, 2013. On secure VANETbased ad dissemination with pragmatic cost and effect control. IEEE Transact. Intell Transport. Syst., 14: 124-135. DOI: 10.1109/TITS.2012.2206111

Lu, R., X. Lin, H.T. Luan, X. Liang and X. Shen, 2012. Pseudonym changing at social spots: An effective strategy for location privacy in VANETs. IEEE Trans. Vehicular Technol., 61: 86-96. DOI: 10.1109/TVT.2011.2162864

Ma, X., J. Zhang, X. Yin and K.S. Trivedi, 2012. Design and analysis of a robust broadcast scheme for vanet safety-related services. IEEE Trans. Vehicular Technol., 61: 46-61. DOI: 10.1109/TVT.2011.2177675

Mershad, K., Lebanon and H. Artail, 2013. A framework for secure and efficient data acquisition in vehicular ad hoc networks. IEEE Trans. Vehicular Technol., 62: 536-551. DOI: 10.1109/TVT.2012.2226613

Pattnaik, O. and B.K. Pattanayak, 2014. Security in vehicular ad hoc network based on intrusion detection system. Am. J. Applied Sci., 11: 337-346. DOI: 10.3844/ajassp.2014.337.346

Ros, F.J., P.M. Ruiz and I. Stojmenovic, 2012. Acknowledgment-based broadcast protocol for reliable and efficient data dissemination in vehicular ad hoc networks. IEEE Trans. Mobile Comput., 11: 33-46. DOI: 10.1109/TMC.2010.253
Samara, G., S. Ramadas, A.H. Wafaa and A. Salihy, 2010. Safety message power transmission control for vehicular ad hoc networks. J. Comput. Sci., 6: 1056-1061. DOI: 10.3844/jcssp.2010.1056.1061

Shim, K.A., 2012. CPAS: An efficient conditional privacy-preserving authentication scheme for vehicular sensor networks. IEEE Trans. Vehicular Technol., 61: 1874-1883. DOI: 10.1109/TVT.2012.2186992

Simo, R.J., Reigadas, A.M. Fernandez, J.R. Lopez and J.S. Pascual, 2010. Modeling and optimizing IEEE 802.11 DCF for long-distance links. IEEE Trans. Mobile Comput., 9: 881-896. DOI: 10.1109/TMC.2010.27

Tseng, F.K., Hsinchu, Taiwan, Y.H. Liu and J.S. Hwu et al., 2011. A secure reed-solomon code incentive scheme for commercial Ad dissemination over VANETs. IEEE Trans. Vehicular Technol., 60: 4598-4608. DOI: 10.1109/TVT.2011.2172471

Zhang, C., X. Lin, R. Lu, P.H. Ho and X. Shen, 2008. An efficient message authentication scheme for vehicular communications. IEEE Trans. Vehicular Technol., 57: 3357-3368. DOI: 10.1109/TVT.2008.928581 